\title{
Memory Impairment in Rats after Desflurane Anesthesia is Age and Dose Dependent
}

\author{
Jennifer K. Callaway ${ }^{\mathrm{a}, *}$, Nigel C. Jones ${ }^{\mathrm{b}}$, Alistair G. Royse ${ }^{\mathrm{c}}$ and Colin F. Royse ${ }^{\mathrm{d}}$ \\ ${ }^{a}$ Department of Pharmacology, University of Melbourne, Melbourne, Victoria, Australia \\ ${ }^{\mathrm{b}}$ Department of Medicine (Royal Melbourne Hospital), Melbourne Brain Centre, University of Melbourne, \\ Melbourne, Victoria, Australia \\ ${ }^{\mathrm{c}}$ Department of Surgery, University of Melbourne; and Cardiothoracic Surgeon, Department of Cardiac Surgery, \\ The Royal Melbourne Hospital, Melbourne, Victoria, Australia \\ ${ }^{\mathrm{d}}$ Department of Surgery, University of Melbourne; and Cardiothoracic Anesthesiologist, The Royal Melbourne \\ Hospital, Melbourne, Victoria, Australia
}

Handling Associate Editor: Pravat Kumar Mandal

\begin{abstract}
Post-operative cognitive dysfunction (POCD) predominantly affects the elderly who suffer memory and concentration deficits after anesthesia and surgery. Animal studies have demonstrated anesthetic alone may contribute to POCD but results are variable and little is known about common anesthetics other than isoflurane. The present study investigated dose-dependence of desflurane anesthesia in young adult and aged rats. We hypothesize higher concentrations of desflurane will result in memory impairment in the water maze and that impairment will be worse in aged rats. Effects of anesthesia ( 1 or $1.5 \mathrm{MAC}, 4 \mathrm{~h})$ desflurane, or sham exposure on cognition were investigated in young adult (3 months) and aged (20-24 months) rats at 1, 4, and 12 weeks post-exposure. The Morris water maze was used to assess acquisition and retention of spatial reference memory. Latency to find the hidden platform and swimming speed were compared between treatments. Aged rats showed significant impairment in task acquisition after exposure to 1.5 MAC, but not 1.0 MAC desflurane anesthetic when tested 1 week following exposure. Latency to find the platform and distance travelled were significantly longer in aged rats given 1.5 MAC desflurane (latency: $\mathrm{F}_{(1,108)}=19.71, p<0.0001$; distance: $\left.\mathrm{F}_{(1,108)}=5.79, p=0.018\right)$. Deficits were not long-lasting and were no longer present at 4 or 12 weeks. In contrast, young adult rats performed equally as well as sham-exposed control rats irrespective of desflurane dose. This study showed the effects of desflurane on learning and memory in the water maze are age and dose dependent and are brief in duration.
\end{abstract}

Keywords: Aging, anesthetics inhalation, cognitive, desflurane, memory disorders, retention disorders

\section{INTRODUCTION}

Post-operative cognitive dysfunction (POCD) is a recognized complication following surgery lasting days to weeks but in some patients, particularly the elderly, POCD can persist for months or can be permanent $[16,22,24]$. The incidence of POCD is greater

*Correspondence to: Jennifer Callaway, PhD, Department of Pharmacology, Level 8, Medical building, The University of Melbourne, Carlton, 3010 Victoria, Australia. Tel.: +61 38344 8304; Fax: +61 38344 0421; E-mail: callaway@unimelb.edu.au. in the elderly and is associated with poor outcome following surgery and increased mortality [23, 24]. The cause of POCD is still under investigation but anesthesia itself has been implicated mainly as a result of studies in experimental animals. But clinical studies have found that the relative risk of mortality increased by $19-34 \%$ as length of deep anesthesia increased in patients undergoing non-cardiac surgery with general anesthesia [23, 24]. Apart from this suggestion that depth of anesthesia could potentially influence POCD, there have been no studies investigating dose-response effects of anesthesia on cognitive outcome. 
Pre-clinical studies designed to investigate the effects of anesthesia alone without the influence of any surgical interventions have generated variable results. In agreement with clinical studies, aged rats have generally been shown to have impaired memory following exposure to isoflurane [7, 8]. Cognitive deficits have been reported in young adult rats exposed to isoflurane $[5,17]$ but improvements have also been noted [6]. Most studies have concentrated on isoflurane which remains a commonly used anesthetic worldwide. There is very little data on the cognitive effects of newer inhalational anesthetics such as sevoflurane and desflurane that is relevant to the memory deficits that occur in POCD namely, impaired cognition weeks or months subsequent to exposure to anesthesia, when the agent is no longer present in the body.

Further implicating anesthesia alone as a possible cause of POCD, pathological studies in experimental animals have demonstrated increased apoptosis, as well as increased pathology associated with Alzheimer's disease following exposure of mice to isoflurane [32], isoflurane plus nitrous oxide [36], sevoflurane [10], and desflurane combined with hypoxia [35]. In these studies, however, no tests of cognition were performed so it is unknown whether such changes result in POCD. In this regard, it has recently been shown in 3 month-old rats that exposure to isoflurane induces caspase- 3 activation, reduced neuronal density and increased interleukin-1 $\beta$ (IL-1 $\beta$ ) in the hippocampus $6 \mathrm{~h}$ post-exposure and also results in hippocampal-dependent memory impairments [17]. No changes in amyloid- $\beta$ (A $\beta$ ) peptide were detected in the cortex at 29 days after exposure when cognitive deficits were present.

We have recently found that 1 minimum alveolar concentration (MAC) sevoflurane, rather than causing cognitive deficits after exposure, improved acquisition learning in the Morris water maze in both young adult and aged rats [5]. Our findings suggest that even if sevoflurane does cause early neuropathology after exposure, these changes may not necessarily lead to cognitive dysfunction. Interestingly, Zhang and coworkers did not find any neuropathological changes following desflurane alone but only when desflurane was combined with hypoxia [35]. Accordingly, desflurane anesthesia may be less likely to cause cognitive deficits than other similar agents. Therefore, in the present study we aimed to investigate the effects of a moderate duration of desflurane $(4 \mathrm{~h})$ at low and high concentrations (1 and 1.5 MAC) in both young adult (3 months) and aged (18-21 months) rats on cognition at 1,4 , and 12 weeks following anesthetic exposure.

\section{MATERIALS AND METHODS}

\section{Anesthesia}

The University of Melbourne Animal Ethics Committee on the use of animals in a research project approved this study. The 93 male Sprague Dawley rats (3 months of age, $n=40$, weighing $500.1 \pm 17.8 \mathrm{~g}$ ), and ex-breeders (18-21 months of age at the commencement of the experiment, $n=31$, weighing $747.9 \pm 17.5 \mathrm{~g}$ ) used in these experiments were sourced from the Animal Resources Centre, Canning Vale, Western Australia. Rats were housed on a $12 \mathrm{~h}$ light-dark cycle in a climate and humidity controlled room with free access to food and water.

Rats were randomly assigned to either sham exposure or desflurane anesthesia groups by drawing treatment condition and a rat number written on folded papers. The experimenter was blinded to treatment condition in water maze testing. Young adult rats were tested at different times several months apart from aged rat cohorts. In each experiment, a separate group of sham rats exposed to oxygen alone was tested alongside the desflurane exposed rats. Rats in the anesthesia groups were exposed to one of two difference doses of desflurane (1 MAC or 1.5 MAC) to assess dose-dependency. MAC was determined for desflurane for young and aged rats as described below. Rats were placed in groups of 3-4 in an induction chamber that was filled with desflurane $(10 \%)$ until unconscious (approximately 1-2 min). Rats were then removed and placed at one of the nose cones of our custom designed anesthetizing apparatus as previously described [4]. Desflurane was administered at a flow rate of approximately $1.5 \mathrm{~L} / \mathrm{min}$ into the anesthetic chamber and gas concentrations were monitored continuously (Ohmeda Excel 210 SE anesthetic machine, Datex Instrumentarium Corp., Helsinki, Finland) and altered to maintain oxygen and carbon dioxide at stable levels. Our preliminary testing of the apparatus found identical anesthetic concentrations at each nose cone for any given flow rate between 1 and $3 \mathrm{~L} / \mathrm{min}$.

Warming mats and measurement of rectal temperatures at $30 \mathrm{~min}$ intervals were employed to maintain normothermia $\left(37 \pm 1^{\circ} \mathrm{C}\right)$. To maintain hydration during the long period of anesthesia, rats were given $0.9 \%$ saline solution $(0.25 \mathrm{ml} / 100 \mathrm{~g}$ body weight/hour $)$ subcutaneously. Blood pressure was determined at hourly intervals during anesthetic exposure using noninvasive tail cuffs (Coda, Kent Scientific, Torrington, CT). Sham rats (no anesthetic exposure) were placed 
in the induction chamber containing $100 \%$ oxygen only for $10 \mathrm{~min}$ and then returned to their home cages.

MAC was formally determined for desflurane in separate groups of rats $(n=12$ young adult, 10 weeks of age, weighing $335.3 \pm 2.2 \mathrm{~g}$ and $n=10$ aged rats, 19 months, $707.3 \pm 5.7 \mathrm{~g}$ ) according to the method described by Barbry and colleagues [3]. Briefly, desflurane was commenced at approximately $80 \%$ of previously determined MAC in rats $(7.97 \% \pm 0.75)$ [13] for $1 \mathrm{~h}$ and then the concentration was increased by $0.5 \%$ every $30 \mathrm{~min}$ and the number of rats moving in response to a forcep clamped to the first ratchet on the tail was noted.

\section{Morris water maze test}

Behavioral testing was performed in a lightcontrolled room in the Behavioral Testing Facility at the Department of Medicine, Royal Melbourne Hospital, The University of Melbourne. Prior to each day of testing, rats were given at least $30 \mathrm{~min}$ to become accustomed to the conditions of the facility. The maze consisted of a $160 \mathrm{~cm}$ diameter black plastic pool filled to a depth of $30 \mathrm{~cm}$ with clear water maintained at $23 \pm 1^{\circ} \mathrm{C}$. For each trial a rat was gently placed in the pool at one of four locations which was changed on each of the four daily trials. Rats were allowed $90 \mathrm{~s}$ to locate a $10 \mathrm{~cm}$ diameter black platform submerged $2 \mathrm{~cm}$ below the surface of the water using visual cues in the room and around the edges of the pool as previously described [25] with modifications [14]. Testing was conducted in a darkened room so the black submerged platform is not visible in the black pool. Each rat was randomly assigned one of four different platform positions, which was kept constant for each individual rat during for the entire test. If the rat was unable to find the platform within the $90 \mathrm{~s}$ trial, it was guided to the platform where it was allowed to remain for $30 \mathrm{~s}$. At the end of each trial rats were towel dried and returned to their home cage. Four trials were conducted per session with an interval of 30 min between trials and $24 \mathrm{~h}$ between each session. The experiment was conducted over four consecutive days, commencing one week after anesthetic exposure. Trials were videotaped and swim path tracked using Ethovision Video-Tracking Software (v3.1.16 Noldus Information Technology, Wageningen, Netherlands). For each trial, latency to reach the platform, total distance traveled, and swim speed were calculated, and averaged over each daily session.
Twenty four hours after acquisition, rats were tested in a probe trial. Each rat is placed in the pool for 90 $\mathrm{s}$ without the platform. Time spent in the quadrant where the platform was previously located is taken as a measure of memory for platform position. Four weeks following anesthetic or sham exposure, a reversal trial was conducted where a new platform position different from the first was assigned to each rat. Rats were then required to learn the new platform location. The reversal trial was conducted over only two daily sessions of four trials since criterion was reached after only two days because rats had already learned the rules of the task, that is, when they sit on the platform they are taken out of the pool. The experimental time line is shown in Fig. 1. Twenty four hours following the reversal trial, rats were subjected to a second probe trial to test memory for the new platform location. Three months post-exposure rats were tested in a third probe trial to assess long-term memory retention, then subjected to another reversal trial (platform location different from week 1 and week 4), and then a fourth and final probe trial $24 \mathrm{~h}$ later.

\section{Statistical analyses}

Estimates of sample size were based on previous data [4] showing a difference in means between two treatment groups of $15.1 \%$ with a pooled standard deviation of $12.5 \%$. A sample size of 10 should enable the detection of at least $15 \%$ difference with a power of $80 \%$ and alpha of $5 \%$, with an assumed standard deviation of $12.5 \%$.

Sigmoidal dose-response curve analysis was used to fit curves of the percentage of rats showing no movement under desflurane exposure (MAC determination). MAC, defined as no movement in $50 \%$ of the animals in response to stimulus, and $95 \%$ confidence intervals (CI) were calculated using GraphPad Prism (GraphPad Software Inc., La Jolla, CA). Percentage inhaled desflurane was analyzed by unpaired Student's $t$-tests. Systolic and diastolic blood pressure in young adult and aged sham and desflurane-treated rats was compared using a 3-way ANOVA with repeated measures on time of determination. Three-way ANOVA with

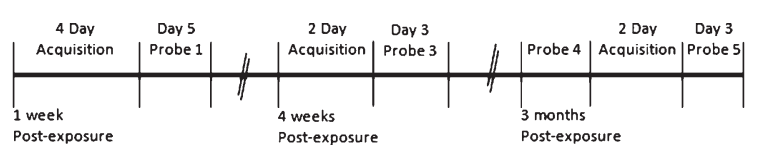

Fig. 1. Experimental timeline for Morris water maze testing showing acquisition trials at 1,4 , and 12 weeks post desflurane exposure and the time at which the five probe trials were conducted. 
factors treatment, dose, and session number, the latter being repeated measures factor, was used for analysis of latency, distance travelled, and velocity in the acquisition phase of the Morris water maze test. Post-hoc testing was done using Tukey's HSD test (equal or unequal $\mathrm{n}$ where appropriate). In the probe trials, the time spent in the target quadrant was analyzed using two-way ANOVA with factors treatment, and probe number as a repeated measures factor. Data were analyzed using GraphPad Prism 5 (GraphPad Software Inc., La Jolla, CA) and Statistica (Statsoft Inc., Tulsa, OK) and in all cases, statistical significance was defined as $p<0.05$.

\section{RESULTS}

\section{Physiological variables}

Of the 31 aged rats, a total of 10 rats died or had to be euthanized during the 13 weeks under study. Four desflurane-treated rats and 5 sham rats died and had significant weight loss with unknown pathology on autopsy. Of the rats that were euthanized, one desflurane-treated rat had a large tumor on the abdomen and one sham rat had hock sores and swollen hind paws.

MAC was determined as to be $8.21 \%$ (95\% CI: 7.99 to $8.43 \%$; Fig. $2 \mathrm{~A})$ in young adult rats and $6.99 \%(95 \%$ CI: 6.69 to $7.29 \%$; Fig. 2A) in aged rats. The MAC values determined from this experiment were used as the 1 and 1.5 MAC concentrations for anesthetic exposures in young and aged rats.

Systolic and diastolic blood pressure remained within normal physiological limits during anesthetic exposure, however high dose desflurane induced significant hypotension. Systolic and diastolic blood pressure remained stable over the $4 \mathrm{~h}$ of exposure to either 1.0 or $1.5 \mathrm{MAC}$ dose desflurane in young adult rats $\left(\mathrm{F}_{(3,118)}=0.04, p=0.99\right.$, Fig. $\left.2 \mathrm{~B}\right)$. Young adult rats had significantly lower systolic and diastolic blood pressure after exposure to high dose desflurane $\left(\mathrm{F}_{(1,118)}=13.28, p=0.0004\right.$, Fig. $\left.2 \mathrm{~B}\right)$. In aged rats, systolic and diastolic blood pressure was significantly lower in the 1.5 MAC desflurane-exposed rats but only during the first $2 \mathrm{~h}$ of exposure (dose $\mathrm{x}$ time interaction: $\mathrm{F}_{(3,85)}=4.73, p=0.0042$, Fig. 2C) after which blood pressure increased significantly in this group. Temperatures were within normal physiological levels throughout desflurane exposure in both young and aged desflurane exposed rats (data not shown).
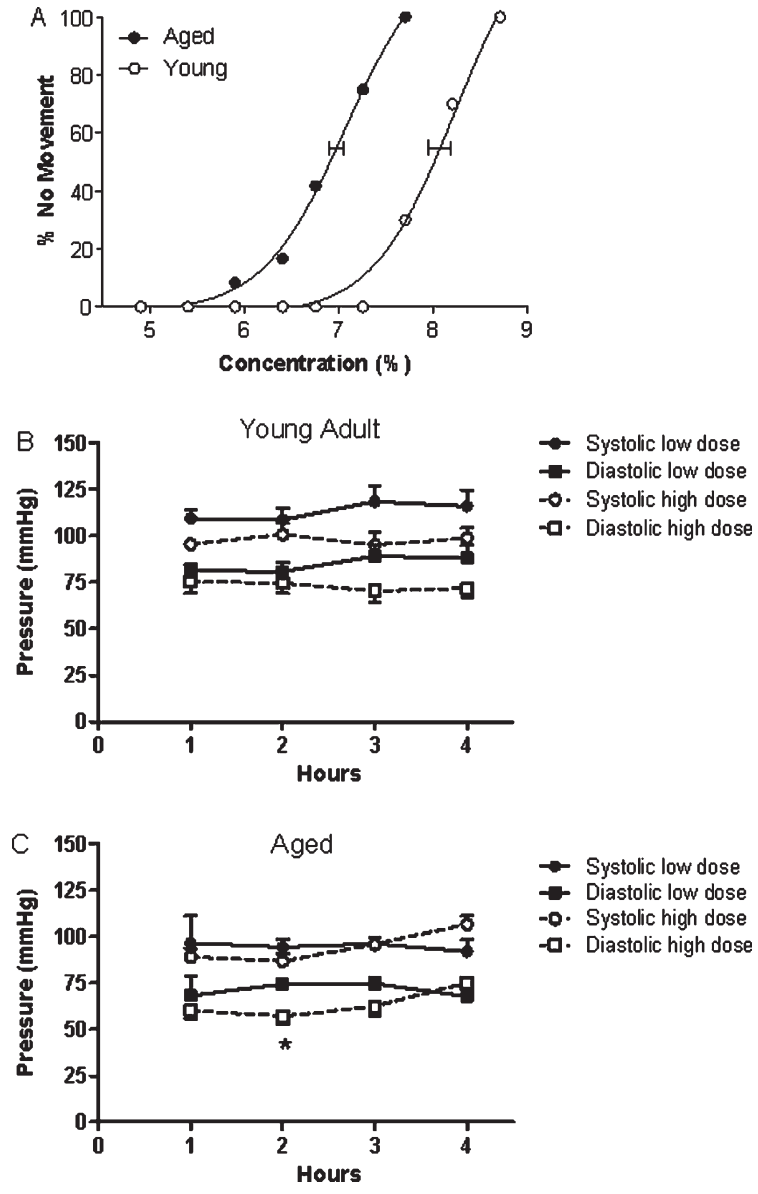

Fig. 2. (A) Percentage of young and aged rats with no movement in response to increasing concentrations of desflurane. Each point represents $N=10-12$ rats. Curves were fitted using sigmoidal concentration-response analysis. The minimum alveolar concentration (MAC) for young adult and aged rats with 95\% CI (horizontal bars) is shown. (B) Systolic and diastolic blood pressure in young adult rats anaesthetized with low dose (closed symbols) or high dose desflurane (open symbols). Data are mean \pm SEM for $N=12$ rats per treatment group. (C) Systolic and diastolic blood pressure in aged rats anaesthetized with low dose (closed symbols) or high dose desflurane (open symbols). Data are mean \pm SEM for $N=10$ rats per treatment group.

\section{Morris water maze testing}

Groups of young adult rats exposed to sham conditions or to 1.0 and 1.5 MAC desflurane anesthesia learned to find the hidden platform in the water maze as evidenced by a significant decrease in latency to locate the platform across the 4 daily sessions (Fig. 3A; $F_{(3,144)}=115.77, p<0.0001$ ). There was no significant treatment or dose effect of exposure to desflurane on latency when young adult rats were tested at 1,4 , or 12 weeks compared with sham- 
exposed young rats (Fig. 3A, C, and E). Aged rats also learned to find the platform and improved in performance over time (Fig. 3B: $\mathrm{F}_{(3,108)}=44.03, p<0.0001$ ). However, in contrast, there was a significant effect of dose on latency to locate the hidden platform in aged rats. Exposure to 1.5 MAC desflurane resulted in aged rats taking significantly longer to find the platform compared with 1.0 MAC desflurane-exposed rats or sham-exposed rats $\left(\mathrm{F}_{(1,108)}=19.71, p<0.0001\right)$. Exposure to 1.0 MAC desflurane had no effect on acquisition of the task compared with corresponding sham controls $(p>0.05)$. The impairment following 1.5 MAC desflurane in latency to locate the hidden platform was no longer evident in the reversal trials at 4 (Fig. 3D: $\mathrm{F}_{(1,50)}=0.002, p=0.962$ ) or 12 weeks (Fig. 3F: $\mathrm{F}_{(1,34)}=0.527, p=0.473$ ).

There were no significant treatment or dose effects on the distance travelled by young adult rats while locating the submerged platform in the maze when tested at 1, 4, or 12 weeks (Fig. 4A, C, and E). A significant effect of dose was found in the speed at which young adult rats swam in the maze 1 week following desflurane exposure (Fig. 5A: $\mathrm{F}_{(1,144)}=11.064$, $p=0.001)$. There was a significant interaction between factors treatment and dose $\left(\mathrm{F}_{(1,144)}=8.24, p=0.005\right)$ with 1.5 MAC desflurane-treated rats swimming significantly faster than 1.0 MAC desflurane-treated rats. Differences in swim speed between doses were not significant at 4 weeks (Fig. 5C, $p>0.05$ ) but a significant interaction between treatment and dose was again found at 12 weeks (Fig. 5E: $\mathrm{F}_{(1,70)}=4.272, p=0.042$ ) where 1.5 MAC desflurane-treated rats swam at a greater velocity.

In aged rats, 1.5 MAC desflurane also significantly affected the distance travelled in the maze (Fig. 4B: $\left.\mathrm{F}_{(1,108)}=5.79, p=0.018\right)$ with these rats travelling further compared with 1.0 MAC desflurane rats. The dose-dependent increases in latency and distance travelled by 1.5 MAC desflurane exposed rats could not be accounted for by differences in swim speed as no significant differences were found in velocity between treatments (Fig. 5A: $\mathrm{F}_{(1,108)}=1.445, p=0.232$ ). Desflurane treatment at either 1.0 or $1.5 \mathrm{MAC}$, had no effect on distance travelled in the maze when aged rats were re-tested in reversal trials at 4 weeks (Fig. 4D; $\mathrm{F}_{(1,50)}=0.435, p=0.512$ ) and 12 weeks $\left(\mathrm{F}_{(1,34)}=0.026, p=0.872\right)$. Likewise desflurane treatment did not affect swim speed at 4 or 12 weeks test times in aged rats (Fig. 5D, F; $p>0.05$ ).

There were no statistically significant differences between sham and 1.0 and 1.5 MAC desfluraneexposed young adults (Fig. 6A and $\mathrm{C}$, respectively) or aged rats (Fig. 4B and D, respectively) on any of the five probe trials conducted over the 12 weeks of testing (see Fig. 1 for probe schedule).

\section{DISCUSSION}

This study investigated dose-dependence and longterm effects of desflurane on cognitive performance in experimental animals. The main finding is that the effects of desflurane on acquisition learning and memory in the water maze are dependent on age and dose. In young adult rats, desflurane had no effect on acquisition of the water maze task or on memory for platform location when rats were tested 1,4 , or 12 weeks after treatment. In aged rats, however, the effects of desflurane were dependent on dose, with the higher dose of 1.5 MAC desflurane exposure resulting in rats taking longer and travelling further when searching for the submerged platform in the water maze. These detrimental effects were not long lasting and by 4 weeks post-exposure 1.0 and 1.5 MAC desflurane-exposed rats performed the same as sham-exposed controls in the reversal trial. Treatment with 1.5 MAC desflurane affected acquisition of the water maze task but did not affect memory for target location as demonstrated by similar preference between sham-exposed and desflurane treated rats for the target quadrant in all probe trials.

Previous studies in rodents have also demonstrated a greater vulnerability of aged rats to cognitive deficits after exposure to isoflurane anesthetic [7-9] and in clinical trials age has been identified as a risk factor for POCD [22]. The mechanism through which anesthesia causes POCD and the reason for greater vulnerability of the elderly is still uncertain. Maze and colleagues have demonstrated that a peripheral inflammatory reaction occurs during surgery triggering a cytokine cascade and inflammation in the hippocampus that can result in impaired cognitive performance $[12,31]$. Lin co-workers [17] have also demonstrated that exposure to isoflurane anesthesia alone can cause a similar inflammatory response with increased levels of the cytokine interleukin- $1 \beta$ accompanied by cognitive deficits. It remains to be tested whether other commonly used anesthetics such as desflurane also induce such an inflammatory response.

Evidence that anesthesia alone may cause POCD has also been suggested by studies in experimental animals demonstrating increased apoptosis, and pathological markers associated with Alzheimer's disease following exposure of mice to various anesthetics $[10,32-36]$. Interestingly, no neuropathological 
Young Adult Rats

A

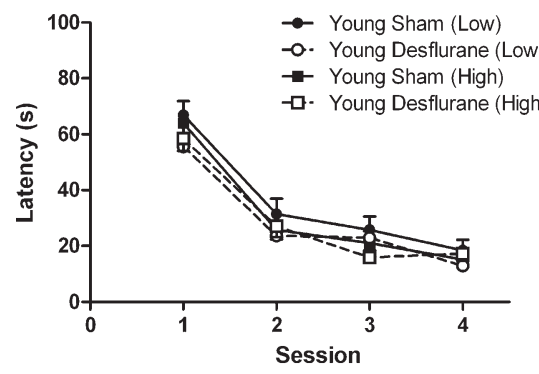

C

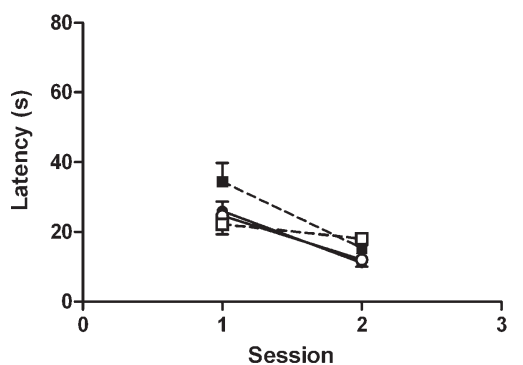

E

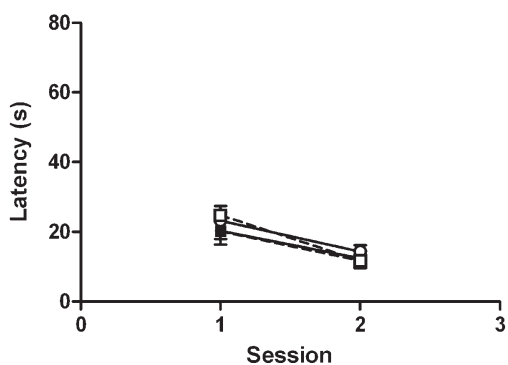

Aged Rats

B

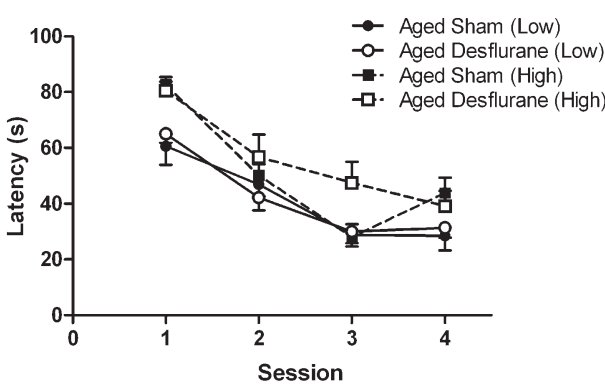

D

4 Weeks

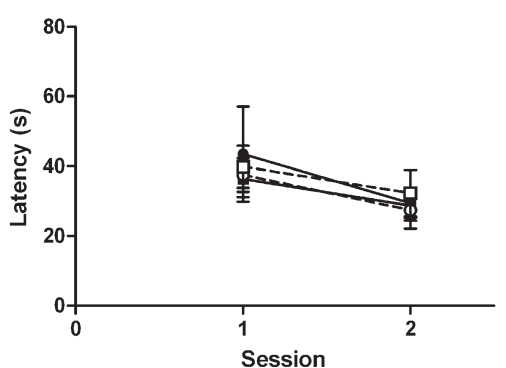

$\mathrm{F}$

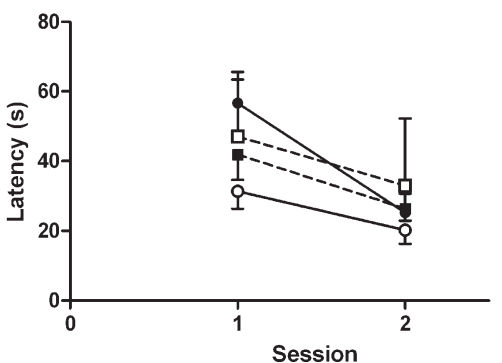

Fig. 3. Effects of $4 \mathrm{~h}$ exposure to 1.0 or $1.5 \mathrm{MAC}$ desflurane in young adult and aged rats on latency to locate the hidden platform in the Morris water maze at (A and B) 1 week, (C and D) 4 weeks, and (E and F) 12 weeks post-exposure. Desflurane-exposed rats performed significantly slower than sham-exposed controls at 1 week in aged (B) but not (A) young adult rats $\left(^{*} p<0.0001\right.$, ANOVAs and Tukey honestly significant difference test). This effect was transient and no treatment or dose effects were apparent at 4 or 12 weeks post desflurane exposure in aged rats (D and F). Young rats, weeks 1, 4 and 12: 1.0 MAC: shams $N=9$, desflurane $N=11 ; 1.5 \mathrm{MAC}$ : sham $N=10$, desflurane $N=10$. Aged rats, 1.0 MAC: Shams $N=5,5$ and 3 and desflurane $N=9,9$ and 8 for weeks 1,4 and 12 respectively; 1.5 MAC: shams $N=8,7$ and 6 and desflurane $N=9,8$ and 4 for weeks 1,4 and 12 respectively. Data are mean \pm SEM.

changes were found following desflurane alone but only when desflurane was combined with hypoxia [35]. These previous studies did not investigate cognitive performance in anesthetic-exposed mice. If desflurane alone does not induce this pathology but can cause cognitive deficits, the present study throws doubt on the significance of early apoptosis and markers associated with Alzheimer's disease as a mechanism in causing POCD.
Mandal and colleagues in studies investigating the biochemical and biophysical characteristics of inhaled and intravenous anesthetics discovered that the molecular size of the anesthetic has a profound effect on the oligomerization process of $A \beta$ [19]. The structural alteration of $A \beta$ which leads to oligomerization is believed to play an important role in Alzheimer's disease [20]. The inhaled anesthetics isoflurane, desflurane, sevoflurane, and halothane are all small 
Young Adult Rats

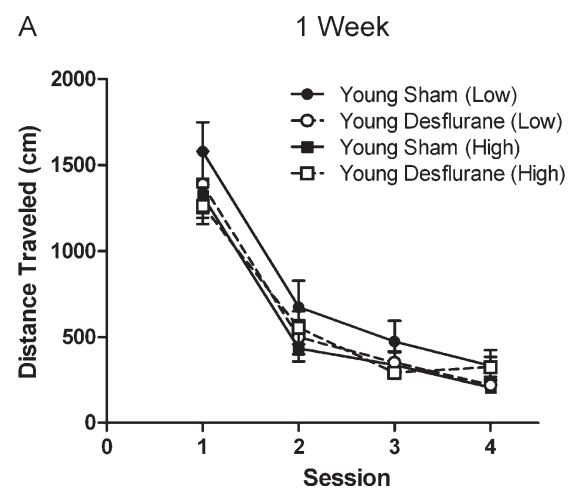

C

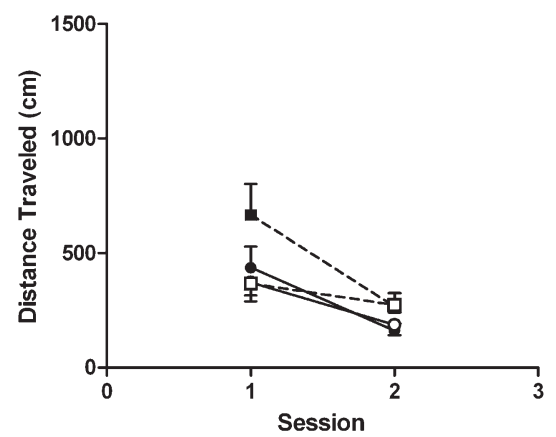

E

12 Weeks

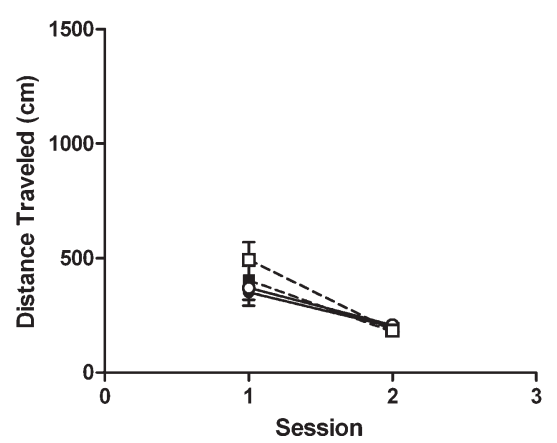

Aged Rats

B

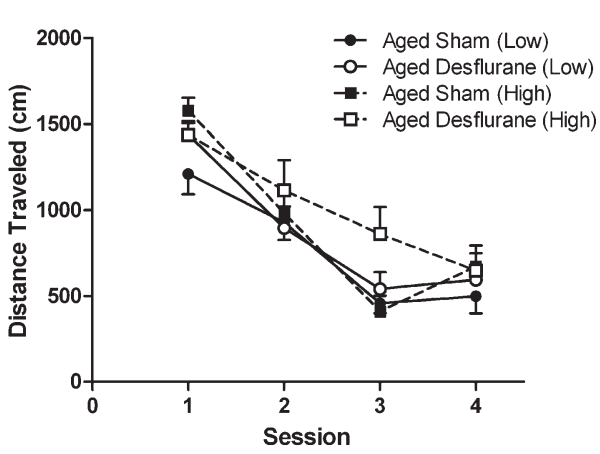

D

4 Weeks

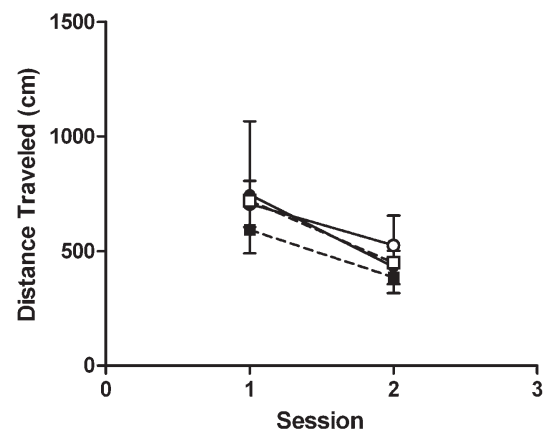

F 12 Weeks

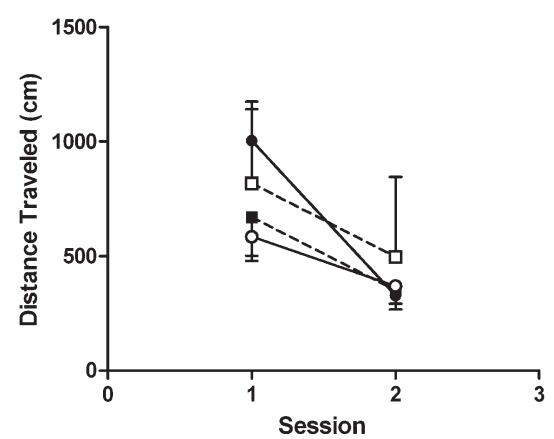

Fig. 4. Effects of $4 \mathrm{~h}$ exposure to desflurane in young adult and aged rats on distance travelled during acquisition in the Morris water maze at (A and B) 1 week, (C and D) 4 weeks, and (E and F) 12 weeks post-exposure. At week 1 aged rats treated with 1.5 MAC desflurane travelled 16 significantly further when searching for the hidden platform in the water maze than 1.0 MAC desflurane-treated rats or sham-exposed controls $(\mathrm{B}, p=0.018)$. This difference was no longer apparent at $4(\mathrm{D})$ or 12 weeks $(\mathrm{F})$. Distance travelled by young adult rats was unaffected by desflurane treatment (A, C and E). Young rats, weeks 1, 4 and 12: 1.0 MAC: shams $N=9$, desflurane $N=11 ; 1.5$ MAC: sham $N=10$, desflurane $N=10$. Aged rats, 1.0 MAC: Shams $N=5,5$ and 3 and desflurane $N=9,9$ and 8 for weeks 1,4 and 12 respectively; 1.5 MAC: shams $N=8,7$ and 6 and dsflurane $N=9,8$ and 4 for weeks 1,4 and 12 respectively. Data are mean \pm SEM.

halogenated molecules [21], whereas propofol and thiopental are large sized molecules. In NMR studies, isoflurane and desflurane led to $A \beta$ oligomerization at concentrations comparable to those used in the intensive care setting after 9 days and 25 days, respectively [19]. The relationship between anesthetic type, $\mathrm{A} \beta$ oligomerization, and cognitive impairment is an intriguing area of investigation. 


\section{Young Adult Rats}

A

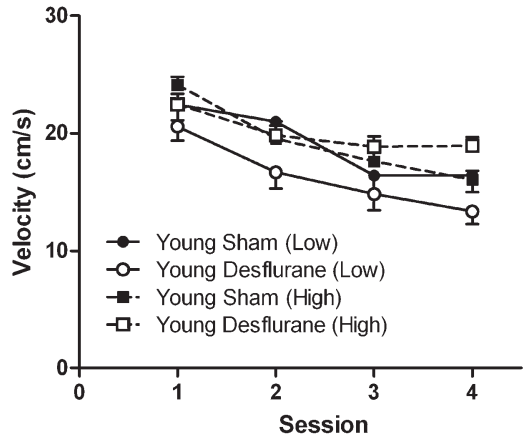

C

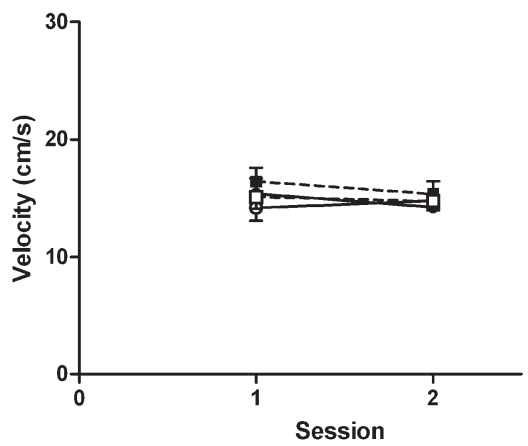

E

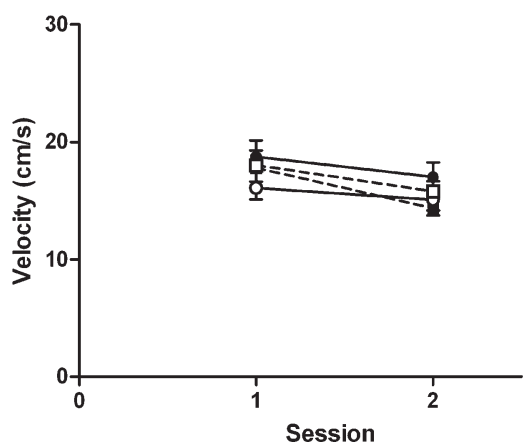

Aged Rats

B

1 Week

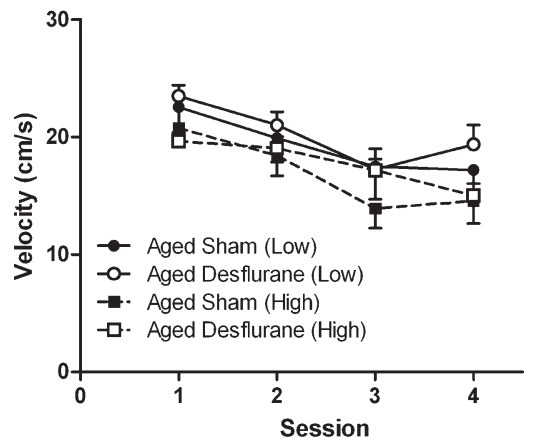

D

4 Weeks

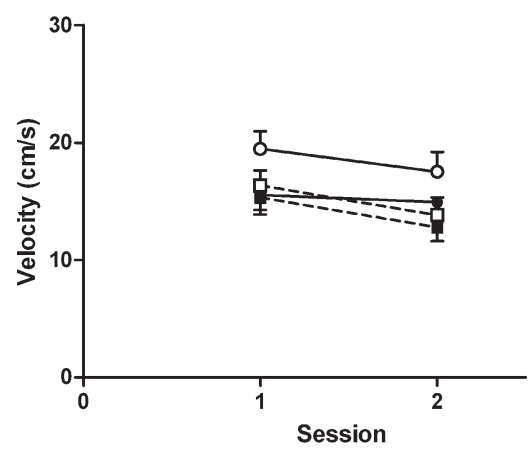

$\mathrm{F}$

12 Weeks

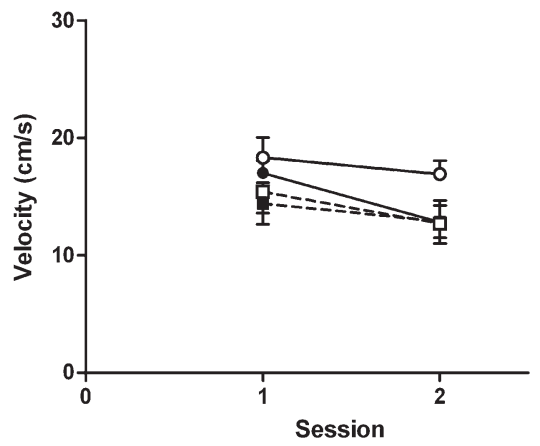

Fig. 5. Effects of $4 \mathrm{~h}$ exposure to desflurane in young adult and aged rats on velocity of swimming during acquisition in the Morris water maze at (A and B) 1 week, (C and D) 4 weeks, and (E and F) 12 weeks post-exposure. Young adult rats exposed to 1.5 MAC desflurane were found to swim significantly faster than $1.0 \mathrm{MAC}$ or sham-exposed controls at 1 week (A; treatment $\mathrm{x}$ dose interaction $P=0.005$ ) and 12 weeks post-exposure ( $\mathrm{E}$; treatment $\mathrm{x}$ dose interaction $P=0.042$ ). There were no significant differences in swim velocity in young adult rats at 4 weeks (C). No treatment or dose effects were evident in aged rats exposed to desflurane compared with controls at any time point. Young rats, weeks 1, 4 and 12: 1.0 MAC: shams $N=9$, desflurane $N=11 ; 1.5$ MAC: sham $N=10$, desflurane $N=10$. Aged rats, 1.0 MAC: Shams $N=5,5$ and 3 and desflurane $N=9,9$ and 8 for weeks 1,4 and 12 respectively; 1.5 MAC: shams $N=8,7$ and 6 and dsflurane $N=9,8$ and 4 for weeks 1,4 and 12 respectively. Data are mean \pm SEM. 
A

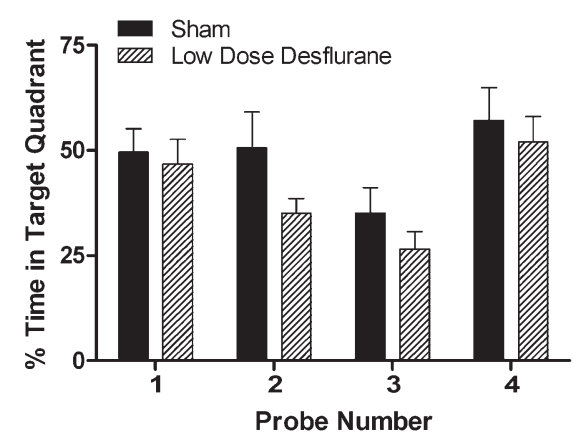

C

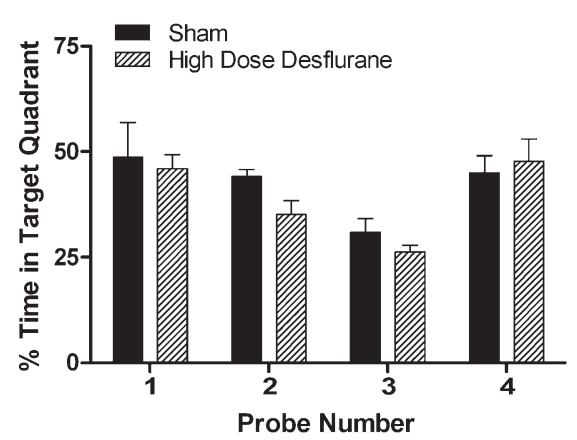

B

Aged Rats

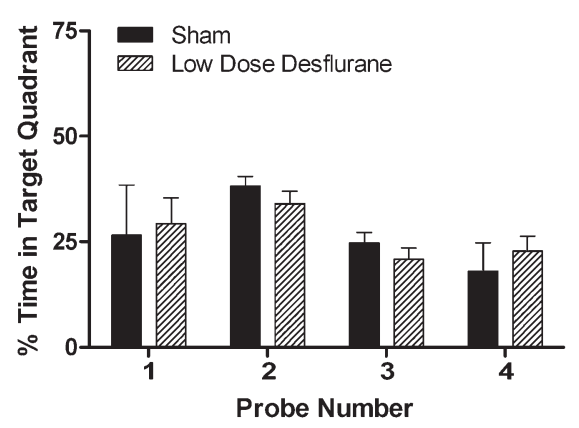

D

Aged Rats

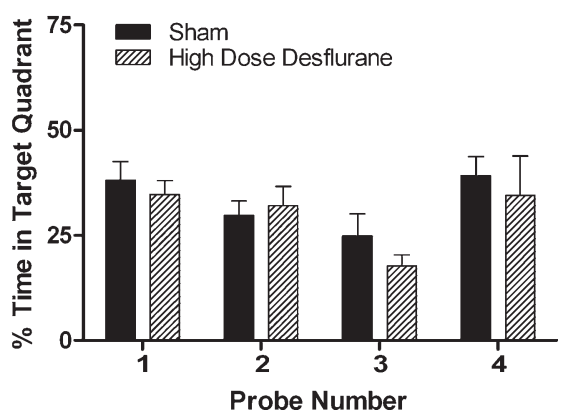

Fig. 6. Probe trials over 12 weeks showing \% time spent in target quadrant by rats exposed to 1.0 and 1.5 MAC desflurane or sham exposure in young rats (A and $\mathrm{C}$, respectively) and aged rats (B and D, respectively). Treatment with desflurane did not affect memory for target platform location at either 1.0 or $1.5 \mathrm{MAC}$ in young adult or aged rats compared with corresponding sham-exposed rats. Young rats, weeks 1 , 4 and 12 : 1.0 MAC: shams $N=9$, desflurane $N=11 ; 1.5$ MAC: sham $N=10$, desflurane $N=10$. Aged rats, 1.0 MAC: Shams $N=5,5$ and 3 and desflurane $N=9,9$ and 8 for weeks 1, 4 and 12 respectively; 1.5 MAC: shams $N=8,7$ and 6 and desflurane $N=9,8$ and 4 for weeks 1,4 and 12 respectively. Data are mean and SEM.

Dose-dependent effects of anesthetics on memory have been reported. Memory retention was inhibited in rats exposed to $0.5,1.0$, and $2 \%$ sevoflurane for $2 \mathrm{~h}$ immediately after inhibitory avoidance training [18]. Administration of low dose sevoflurane $(0.11 \%)$ enhanced memory retention $24 \mathrm{~h}$ later in rats when given during single trial inhibitory avoidance training [1]. Overall these data suggest a dose-dependent effect of anesthesia on memory where low doses enhance and high doses inhibit memory. A dose-dependent effect is supported by in vitro studies showing sub-anesthetic concentrations of sevoflurane enhance LTP and postsynaptic excitatory transmission in hippocampal CA1 region whereas presynaptic inhibition is observed at anesthetic concentrations [26, 28]. However, studies in mouse brain slices indicate that there may be quite a narrow margin between inhibition and enhancement [28]. While these studies offer some insight into the potential mechanisms of action of anesthetics during actual exposure, the clinical picture of POCD differs as cognitive deficits manifest many days, weeks, or months after exposure to anesthetic when pharmacokinetics predict the anesthetic is no longer in the body.

Similar to aged humans [30], the aged rats in our study required significantly less desflurane to induce an equivalent depth of anesthesia to suppress movement to deep paw pinch than the young rats. In aged rats exposed to the high dose of desflurane systolic blood pressure significantly increased in the third and fourth hours of anesthesia. Mean blood pressure has been reported to be higher in aged compared with adult rats [2]. Importantly, in the aged rats, 1.5 MAC of desflurane did not lead to hypotension (systolic blood pressure increased), negating cerebral hypoperfusion as a mechanism for the poorer acquisition memory at 1 week post exposure.

Increased depth of general anesthesia has been linked to higher mortality and to risk of POCD [23, 24]. Clinical trials investigating cognitive outcome after surgery where anesthetic depth is controlled using 
bispectral index have, however, yielded mixed results $[11,15,29]$ and neuromonitoring of anesthesia and POCD remains controversial. In 1,155 patients, Radtke et al. [27] found no influence of intraoperative BIS monitoring on the incidence of POCD, but the percentage of episodes of deep anesthesia was predictive for postoperative delirium. Most of the studies have not investigated specific anesthetic agents which could potentially account for the mixed findings.

A limitation of this study is the small concentration range of anesthetic exposure employed. The 1.5 MAC concentration is not a particularly high concentration of desflurane and hence it is possible that young adult rats could be affected by higher doses. Nevertheless, 1.5 MAC was enough to cause a significant cognitive impairment in the aged rats. Further studies are required to address the mechanism involved in desflurane-induced memory impairment.

We recently reported that sevoflurane (1 MAC, $4 \mathrm{~h}$ ) improved acquisition learning in the water maze in both young and aged rats [4], whereas an equivalent dose and duration of isoflurane impaired memory for platform location in young adult rats under identical experimental conditions to the present study [5]. Together with the present results, and in conclusion, these experiments suggest that even at equivalent concentrations the effects of anesthetics on learning and memory are dependent on anesthetic type and age of the recipient and, as the current study indicates, are also dose-dependent. Our findings of significant memory impairment with high concentration desflurane but not with the equivalent concentration of sevoflurane may inform clinical anesthetic choice for elderly patients in the future.

\section{ACKNOWLEDGMENTS}

This work was supported by grants from The National Heart Foundation, Melbourne, Victoria, Australia (grant \# 809274) and Dementia Seed Funding, National Health and Medical Research Council, Australia (grant \#1028835).

Authors' disclosures available online (http://www.jalz.com/disclosures/view.php?id=2588).

\section{REFERENCES}

[1] Alkire MT, Nathan SV (2005) Does the amygdala mediate anesthetic-induced amnesia? Basolateral amygdala lesions block sevoflurane-induced amnesia. Anesthesiology 102, 754760 .

[2] Anishchenko TG, Semyachkina-Glushkovskaya OV, Berdnikova VA, Sindyakova TA (2010) Effect of age and sex on blood pressure, development of renal hypertension, and con- centrationof nitric oxide in the blood of albino rats. Bull Exp Biol Med 149, 1-3.

[3] Barbry T, Le Guen M, De Castro V, Coriat P, Riou B, Vivien B (2007) Minimum alveolar concentration of halogenated volatile anaesthetics in left ventricular hypertrophy and congestive heart failure in rats. Br J Anaesth 99, 787-793.

[4] Callaway JK, Jones NC, Royse AG, Royse CF (2012) Sevoflurane anesthesia does not impair acquisition learning or memory in the Morris water maze in young adult and aged rats. Anesthesiology 117, 1091-1101.

[5] Callaway JK, Jones NC, Royse CF (2012) Isoflurane induces cognitive deficits in the morris water maze task in rats. Eur $J$ Anaesthesiol 29, 239-245.

[6] Crosby C, Culley DJ, Baxter MG, Yukhananov R, Crosby G (2005) Spatial memory performance 2 weeks after general anesthesia in adult rats. Anesth Analg 101, 1389-1392.

[7] Culley DJ, Baxter M, Yukhananov R, Crosby G (2003) The memory effects of general anesthesia persist for weeks in young and aged rats. Anesth Analg 96, 1004-1009.

[8] Culley DJ, Baxter MG, Crosby CA, Yukhananov R, Crosby G (2004) Impaired acquisition of spatial memory 2 weeks after isoflurane and isoflurane-nitrous oxide anesthesia in aged rats. Anesth Analg 99, 1393-1397.

[9] Culley DJ, Baxter MG, Yukhananov R, Crosby G (2003) Lasting impairment of spatial memory acquisition after general anesthesia in aged rats. J Am Geriatr Soc 51, 571.

[10] Dong YL, Zhang GH, Zhang B, Moir RD, Xia WM, Marcantonio ER, Culley DJ, Crosby G, Tanzi RE, Xie ZC (2009) The common inhalational anesthetic sevoflurane induces apoptosis and increases beta-amyloid protein levels. Arch Neurol 66, 620-631.

[11] Farag E, Chelune GJ, Schubert A, Mascha EJ (2006) Is depth of anesthesia, as assessed by the bispectral index, related to postoperative cognitive dysfunction and recovery? Anesth Analg 103, 633-640.

[12] Fidalgo AR, Cibelli M, White JPM, Nagy I, Maze M, Ma DQ (2011) Systemic inflammation enhances surgery-induced cognitive dysfunction in mice. Neurosci Lett 498, 63-66.

[13] Gong D, Fang Z, Ionescu P, Laster MJ, Terrell RC, Erger EI (1998) Rat strain minimillay influences anesthetic and convulsant requirements of inhaled compounds in rats. Anesth Analg 87, 963-966.

[14] Jones NC, Kumar G, O'Brien TJ, Morris MJ, Rees SM, Salzberg MR (2009) Anxiolytic effects of rapid amygdala kindling, and the influence of early life experience in rats. Behav Brain Res 203, 81-87.

[15] Kerssens C, Gaither JR, Sebel PS (2009) Preserved memory function during bispectral index-guided anesthesia with sevoflurane for major orthopedic surgery. Anesthesiology 111, 518-524.

[16] Knipp SC, Matatko N, Wilhelm H, Schlamann M, Thielmann M, Lösch c, Diener HC, Jakob H (2008) Cognitive outcomes three years after coronary artery bypass surgery: Relation to diffusion-weighted magnetic resonance imaging. Ann Thorac Surg 85, 872-879.

[17] Lin DW, Zhiyi Z (2011) Isoflurane induces hippocampal cell injury and cognitive impairments in adult rats. Neuropharmacology 61, 1354-1359.

[18] Liu XS, Xue QS, Zeng QW, Li QA, Liu JA, Feng XM, Yu BW (2010) Sevoflurane impairs memory consolidation in rats, possibly through inhibiting phosphorylation of glycogen synthase kinase-3 beta in the hippocampus. Neurobiol Learn Mem 94, 461-467.

[19] Mandal PK, Fodale V (2009) Smaller molecular-sized anaesthetics oligomerize a beta peptide simulating alzheimer's 
disease: A relevant issue. Eur $J$ Anaesthesiol 26, 805-806.

[20] Mandal PK, Pettegrew JW (2008) A beta peptide interactions with isoflurane, propofol, thiopental, and combined thiopental with halothane: An nmr study. Biochim Biophys Acta 1778, 2633-2639.

[21] Mandal PK, Pettegrew JW (2008) Clinically relevant concentration determination of inhaled anesthetics (halothane, isoflurane, sevoflurane, and desflurane) by f-19 nmr. Cell Biochem Biophys 52, 31-35.

[22] Moller JT, Cluitmans P, Rasmussen LS, Houx P, Rasmussen H, Canet J, Rabbitt P, Jolles J, Larsen K, Hanning CD, Langeron O, Johnson T, Lauven PM, Kristensen PA, Biedler A, van Beem H, Fraidakis O, Silverstein JH, Beneken JEW, Gravenstein JS, Investigators I (1998) Long-term postoperative cognitive dysfunction in the elderly: Ispocd1 study. Lancet 351, 857-861.

[23] Monk TG, Weldon BC (2010) Anesthetic depth is a predictor of mortality. It's time to take the next step. Anesthesiology 112, 1070-1072.

[24] Monk TG, Weldon BC, Garvan CW, Dede DE, van der Aa MT, Heilman KM, Gravenstein JS (2008) Predictors of cognitive dysfunction after major noncardiac surgery. Anesthesiology 108, 18-30.

[25] Morris R (1984) Developments of water-maze procedure for studying spatial learning in the rat. J Neurosci Methods 11, 47-60.

[26] Otsubo T, Maekawa M, Nagai T, Sakio H, Hori Y (2008) Facilitatory effects of subanesthetic sevoflurane on excitatory synaptic transmission and synaptic plasticity in the mouse hippocampal cal area. Brain Res 1197, 32-39.

[27] Radtke FM, Frank MG, Lendner J, Kruger S, Wernecke KD, Spies CD (2013) Monitoring depth of anaesthesia in a randomized trial decreases the rate of postoperative delirium but not postoperative cognitive dysfunction. Br J Anaesth 110, i98-i105.

[28] Rammes G, Starker LK, Haseneder R, Berkmann J, Plack A, Zieglg Ãnsberger W, Ohl F, Kochs EF, Blobner M (2009)
Isoflurane anaesthesia reversibly improves cognitive function and long-term potentiation (ltp) via an up-regulation in nmda receptor $2 \mathrm{~b}$ subunit expression. Neuropharmacology 56, 626-636.

[29] Steinmetz J, Funder KS, Dahl BT, Rasmussen LS (2010) Depth of anaesthesia and post-operative cognitive dysfunction. Acta Anaesthesiol Scand 54, 162-168.

[30] Steinmetz J, Rasmussen LS (2010) The elderly and general anesthesia. Minerva Anestesiol 76, 745-752.

[31] Terrando N, Monaco C, Foxwell BM, Feldmann M, Maze M (2010) Tumor necrosis factor-alpha (TNF) triggers a cytokine cascade yielding postoperative cognitive decline. Immunology 131, 36-36.

[32] Xie ZC, Culley DJ, Dong YL, Zhang GH, Zhang B, Moir RD, Frosch MP, Crosby G, Tanzi RE (2008) The common inhalation anesthetic isoflurane induces caspase activation and increases amyloid beta-protein level in vivo. Ann Neurol 64, 618-627.

[33] Xie ZC, Dong YL, Maeda U, Moir R, Inouye SK, Culley DJ, Crosby G, Tanzi RE (2006) Isoflurane-induced apoptosis: A potential pathogenic link between delirium and dementia. J Gerontol A Biol Sci Med Sci 61, 1300-1306.

[34] Xie ZC, Dong YL, Maeda U, Moir RD, Xia WM, Culley DJ, Crosby G, Tanzi RE (2007) The inhalation anesthetic isoflurane induces a vicious cycle of apoptosis and amyloid beta-protein accumulation. $J$ Neurosci 27, 1247-1254.

[35] Zhang B, Dong YL, Zhang GH, Moir RD, Xia WM, Yue Y, Tian M, Culley DJ, Crosby G, Tanzi RE, Xie ZC (2008) The inhalation anesthetic desflurane induces caspase activation and increases amyloid beta-protein levels under hypoxic conditions. J Biol Chem 283, 11866-11875.

[36] Zhen Y, Dong Y, Wu X, Xu Z, Lu Y, Zhang Y, Norton D, Tian M, Li S, Xie Z (2009) Nitrous oxide plus isoflurane induces apoptosis and increases beta-amyloid protein levels. Anesthesiology 111, 741-752. 